\title{
The Risks Versus the Benefits of Pharmacologic Treatments, and the Impact of Medical and Psychiatric Comorbidity on Course and Functional Status of Neuropsychiatric Illness
}

\author{
By Eric Hollander, MD
}

This month's CNS Spectrums addresses the risks versus the benefits of pharmacologic treatments and the impacts of medical and psychiatric comorbidity on functional status and course of illness in selected neuropsychiatric disorders.

MatthewT. Huska, MD, and colleagues review the first case report of serotonin syndrome to develop on the highly potent and selective serotonin reuptake inhibitor escitalopram. This occurred in a 24-year-old woman treated with escitalopram $30 \mathrm{mg} /$ day. The clinical features of serotonin syndrome and the impact of polypharmacy on the development of this serious and potentially life-threatening side effect are highlighted. Selective serotonin reuptake inhibitors have a broad spectrum of efficacy in a range of serious and disabling disorders, yet the potential for rare but serious side effects, such as serotonin syndrome, ultimately need to be balanced against the benefits of such treatments in our patients.

James W. Tetrud, MD, notes that levodopa remains the single most effective symptomatic treatment for Parkinson's disease over the past 40 years. However, the response to levodopa therapy changes over time, and its long-term use is often associated with disabling motor complications. This article reviews the basis for these motor complications and highlights their impact on patients' clinical function and quality of life. A risk versus a benefit decision-mak- ing process in the management of Parkinson's disease balances short-term symptom control with long-term functional outcomes, and attempts to minimize motor complications during long-term therapy.

While our field may be moving toward the development of algorithms, such as the Texas Medication Algorithm Project, the practice of both psychiatry and neurology in the treatment of specific neuropsychiatric disorders is ultimately humane, balancing the benefits and the risks of out treatments with our understanding of the individual makeup and goals of our patients.

Stephen I. Deutsch, MD, PhD, and colleagues present the case of a woman with a history of an eating disorder that preceded the diagnosis of a mitochondrial myopathy. It is possible that her eating disorder resulted from a basic defect in mitochondrial oxidative metabolism. If this were so, then direct clinical implications include the idea that mitochondrial myopathies may have variable phenotypic presentations, and that drugs that interfere with mitochondrial oxidative respiration might increase the risk of eating disorders.

Finally, Ana Cristina Gargona Nakata, MD, and colleagues describe the relationship between body dysmorphic disorder (BDD) and obsessive-compulsive disorder (OCD), and their inclusion among the obsessive-compulsive related disorders. The authors examined the

Dr. Hollander is the editor of this journal, Esther and Joseph Klingenstein Professor and Chairman of Psychiatry at the Mount Sinai School of Medicine, and director of the Seaver and New York Autism Center of Excellence in New York City. 
content of preoccupations and level of insight in 103 OCD patients with and without BDD. Patients with comorbid BDD had worse level of insight, lower educational level, more psychiatric comorbidity, and greater presence of somatic and hoarding obsessions, even after adjusting for possible confounders. Within the obsessive-compulsive related disorder, we might conceptualize that different disorders might be characterized by varying severity of associated symptom domains, such as poor insight. These associated symptom dimensions may have a marked impact on phenomenology, course of illness, and response to treatment. Such an alternative dimensional classification schema is being considered for inclusion in the appendix of the Diagnostic and Statistical Manual of Mental Disorders, Fifth Edition.

It is important to remember that while neuropsychiatric disorders may be rooted in dysfunction of brain-based systems, their underlying etiology and pathophysiology may influence the functioning of other organs and systems throughout the body, and that various medical illnesses may manifest with neuropsychiatric presentations. CNS

\section{i3 DLN is now}

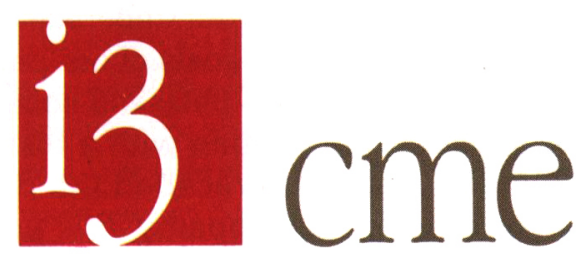

For more information on our

range of evidence-based educational

activities, visit us online at

www.i3cme.com or

call 1-800-326-9166.

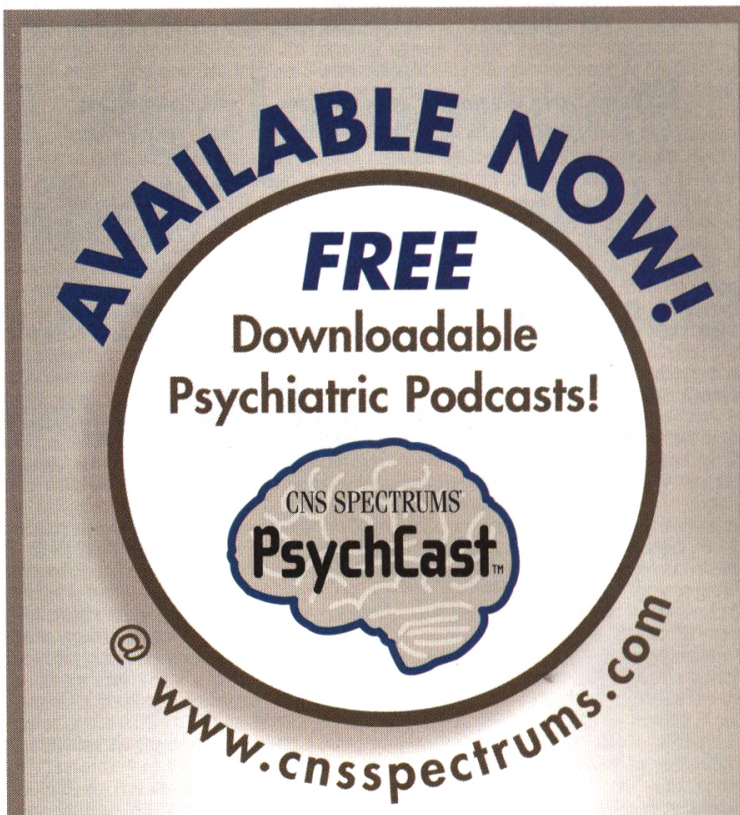

PsychCast ${ }^{\top M}$ is designed to enable physicians to simply and easily listen to a compilation of insightful, 20-35 minute interviews with internationally recognized researchers and clinicians in the fields of psychiatry and neuropsychiatry. Additional 2007 PsychCast $^{\top M}$ programming will include peer-reviewed expert panel dialogues, casebased psychopharmacology lessons, and other educational programs designed to provide our 115,000+ monthly readers with an additional level of personalization in the era of multi-channel learning.

Charles H. Kellner, MD, on the topic of "Current Developments in Electroconvulsive Therapy"

Andrew A. Nierenberg, MD, on the topic of "The Sequenced Treatment Alternatives to Relieve Depression Study: Recent Results"

Richard H. Weisler, MD, on the topic of "Treatment of Attention-Deficit/ Hyperactivity Disorder"

J. Craig Nelson, MD, on the topic of "Treating Late-Life Depression"

Please E-mail us at info@mblcommunications.com to recommend clinical topics you would like to learn about via PsychCast ${ }^{\top M}$ 